\title{
Lipid insertion enables targeted functionalization of paclitaxel-loaded erythrocyte membrane nanosystem by tumor-penetrating bispecific recombinant protein
}

This article was published in the following Dove Press journal:

International Journal of Nanomedicine

\section{Hong Chen ${ }^{1-3, *}$ \\ Huizi Sha ${ }^{2, *}$ \\ Lianru Zhang ${ }^{2}$ \\ Hanqing Qian ${ }^{2}$ \\ Fangjun Chen ${ }^{2}$ \\ Naiqin Ding ${ }^{2}$ \\ Liulian $\mathrm{Ji}^{2}$ \\ Anqing Zhu ${ }^{2}$ \\ Qiuping $X u^{2}$ \\ Fanyan Meng ${ }^{2}$ \\ Lixia Yu ${ }^{2}$ \\ Yan Zhou ${ }^{3}$ \\ Baorui Liu',2}

'The Comprehensive Cancer Centre, Nanjing Drum Tower Hospital Clinical College of Nanjing Medical University, Nanjing, People's Republic of China;

${ }^{2}$ The Comprehensive Cancer Centre of Drum Tower Hospital, Medical School of Nanjing University \& Clinical Cancer Institute of Nanjing University, Nanjing, People's Republic of China; ${ }^{3}$ Department of Oncology, Yixing People's Hospital, Jiangsu, People's Republic of China

*These authors contributed equally to this work

Correspondence: Yan Zhou; Baorui Liu The Comprehensive Cancer Centre of Drum Tower Hospital, Medical School of Nanjing University \& Clinical Cancer Institute of Nanjing University, 32I Zhongshan Road, Nanjing 210008, People's Republic of China

Tel +86 2583107081

$\mathrm{Fax}+862583317016$

Email staffI595@yxph.com; baoruiliu@nju.edu.cn
Background: There is currently much interest in cancer cell targeting and tumor penetrating for research and therapeutic purposes.

Purpose: To improve targeting delivery of antitumor drugs to gastric cancer, in this study, a tumor-targeting biocompatible drug delivery system derived from erythrocyte membrane for delivering paclitaxel (PTX) was constructed.

Methods: Erythrocyte membrane of human red blood cells (RBCs) were used for preparing of erythrocyte membrane-derived vesicles. 1,2-distearoyl-sn-glycero-3-phosphoethanolamine- $N$ (maleimide[polyethylene glycol]-3400) (DSPE-PEG-MAL), a phospholipid derivative, was used to insert tumor-targeting molecular into erythrocyte membrane-derived vesicles. A lipid insertion method was used to functionalize these vesicles without the need for direct chemical conjugation. Furthermore, a tumor-penetrating bispecific recombinant protein named anti-EGFR-iRGD was used for the first time in this work to enable nanosystem to target and penetrate efficiently into the tumor site.

Results: Paclitaxel (PTX)-loaded anti-EGFR-iRGD-modified erythrocyte membrane nanosystem (anti-EGFR-iRGD-RBCm-PTX, abbreviated to PRP) were manufactured. PRP was spheroid, uniformly size, about $171.7 \pm 4.7 \mathrm{~nm}$ in average, could be stable in vitro for 8 days, and released PTX in a biphasic pattern. PRP showed comparable cytotoxicity toward human gastric cancer cells in vitro. In vivo studies showed that, PRP accumulated in tumor site within $2 \mathrm{~h}$ of administration, lasted longer than $48 \mathrm{~h}$, and the tumor volume was reduced $61 \%$ by PRP treatment in Balb/c nude mice, without causing severe side effects.

Conclusion: PRP has potential applications in cancer treatment and as an adjunct for other anticancer strategies.

Keywords: tumor-penetrating bispecific recombinant protein, erythrocyte membrane, biomimetic nanoparticle, drug delivery

\section{Introduction}

Gastric cancer (GC) is one of the leading causes of cancer-related deaths worldwide and is among the top three most commonly diagnosed invasive cancers in the People's Republic of China. ${ }^{1}$ In 2015, the incidence of GC in the People's Republic of China was $\sim 679.1$ per $100,000,{ }^{1}$ considering that the People's Republic of China has a large population size and the burden of the disease is significant. Moreover, most GC cases are diagnosed at an advanced stage, as patients tend to be asymptomatic in the early stage. Chemotherapy remains the mainstay for the treatment of GC; however, based on the therapeutic data, it has been failing to meet clinical expectations. 
Traditional chemotherapy drugs are not tumor specific and trend to spread evenly through the whole body, ${ }^{2,3}$ resulting in mild-to-severe side effects. Paclitaxel (PTX), a commonly used antineoplastic agent, has been proven effective against various solid tumor types ${ }^{4,5}$ and now is one of the firstline drugs for GC. However, the poor aqueous solubility of PTX $(\sim 0.3 \mu \mathrm{g} / \mathrm{mL})$ is one of its major drawbacks; moreover, polyoxyethylated castor oil, the currently used formulation vehicle for the commercially available injectable form of PTX $\left(\right.$ Taxol $\left.^{\circledR}\right)$, has been reported to contribute to the risk of infection and hypersensitivity reactions. ${ }^{6}$ An actively tumortargeting, biocompatible, biodegradable delivery system for PTX would be a promising step toward improving the efficiency of chemotherapy.

Reduction in toxic side effects and improvement in tumor-targeting and killing properties of anticancer drugs are important challenges that must be addressed. To overcome these obstacles, various drug delivery systems have been designed, including different kinds of nanoparticles and biomimetic systems. ${ }^{7-11}$ With the development of nanotechnology, cell membrane-coated nanosystems, which are simple and effective biomimetics, have elicited considerable interest. Erythrocyte membranes have several unique advantages that make them suitable for use as drug carriers 1) red blood cells (RBCs) are abundant, contain no organelles, form double-concave cells in the circulation, can be easily isolated from the body, and provides a sufficient source of membrane; 2) RBCs can stably circulate in the bloodstream for $\sim 4$ months, previous studies have shown that RBC membrane ( $\mathrm{RBCm}$ )-coated delivery vehicles also have long blood circulation time compared with noncoated vehicle controls, ${ }^{9,12}$ and the coated $\mathrm{RBCm}$ is biologically nontoxic and degradable; and 3) erythrocyte membranes provide inherent membrane surface molecules, ${ }^{12-15}$ which endow nanoparticles with the ability to escape from the immune system, ${ }^{15-17}$ different from surface modifications with polymers, such as commonly used poly(ethylene glycol) (PEG), which may lead to a certain degree of immune response and accelerate blood clearance. ${ }^{18-21}$

There is emerging demand for RBC-mimicking and RBCm-based drug delivery systems that are capable of multitasking applications, including active targeting, immunomodulation, and signal stimulation, especially for cancer treatment where there is a need to improve treatment efficiency and minimize off-target side effets. ${ }^{22-24}$ However, RBC-mimicking and RBCm-based drug delivery systems do not necessarily naturally exhibit all these functions and RBCmimicking systems also have a limited ability to target solid tumors. ${ }^{25,26}$ To improve the active targeting ability of these nanosystems, several nongenetic engineering techniques to modify membranes of live cells have been developed. ${ }^{27}$ These include the lipid insertion method reported by Fang et al. ${ }^{28}$ This method relies on physical attachment vis insertion of the lipid portions of biomolecules into the lipid bilayers of membranes; it is convenient and simple and does not require exposure of the biomembranes to chemical reactions, which helps to retain the natural membrane proteins and the integrity of the cell membranes.

Here, we report a novel tumor-targeting delivery system for PTX. This nanosystem uses RBCm-derived microvesicles as carriers for PTX, with anti-EGFR-iRGD, a recombinant protein that was designed and manufactured by our team, ${ }^{29,30}$ as a tumor-targeting molecule. The manufacturing procedure for this nanosystem is illustrated in Figure 1. In the present study, the delivery system was tested for its tumor-targeting ability, its tumor-suppressive function, and its biocompatibility in vitro and in vivo.

\section{Materials and methods Materials}

1,2-Distearoyl-sn-glycero-3-phosphoethanolamine- $N$ [maleimide(polyethylene glycol)-3400] (DSPE-PEG-MAL) was purchased from Nanocs Inc. (New York, NY, USA). PTX was obtained Jiangsu Heng Rui Medicine Co., Ltd. (Nanjing, the People's Republic of China). Near-infrared lipophilic carbocyanine dye $1,1^{\prime}$-dioctadecyltetramethyl indotricarbocyanine iodide (DiR), 3,3'-dioctadecyloxacarbocyanine perchlorate (DiO), and DAPI staining solution were purchased from Beyotime Institute of Biotechnology (Shanghai, the People's Republic of China). Anti-6× His tag antibody (Dylight $^{\circledR}$ 650) (1:1,000) was obtained from Abcam Inc. (Shanghai, the People's Republic of China). Cell culture media Roswell Park Memorial Institute 1640 medium 1640 were purchased from Wisent Inc. (Nanjing, the People's Republic of China), and fetal bovine serum (FBS) was obtained from (Gibco, Carlsbad, CA, USA). The water used in this study was obtained from a Milli-Q Water Purification System (Merck Millipore, Billerica, MA, USA). All other reagents used were of analytical grade.

\section{Cells}

MKN45 cell line was obtained from Chinese Academy of Sciences Cells Bank (Shanghai, the People's Republic of China). The cells were grown in RPMI 1640 supplemented with $10 \% \mathrm{FBS}$ at $37^{\circ} \mathrm{C}$ and $5 \% \mathrm{CO}_{2}$ in a humidified incubator. 

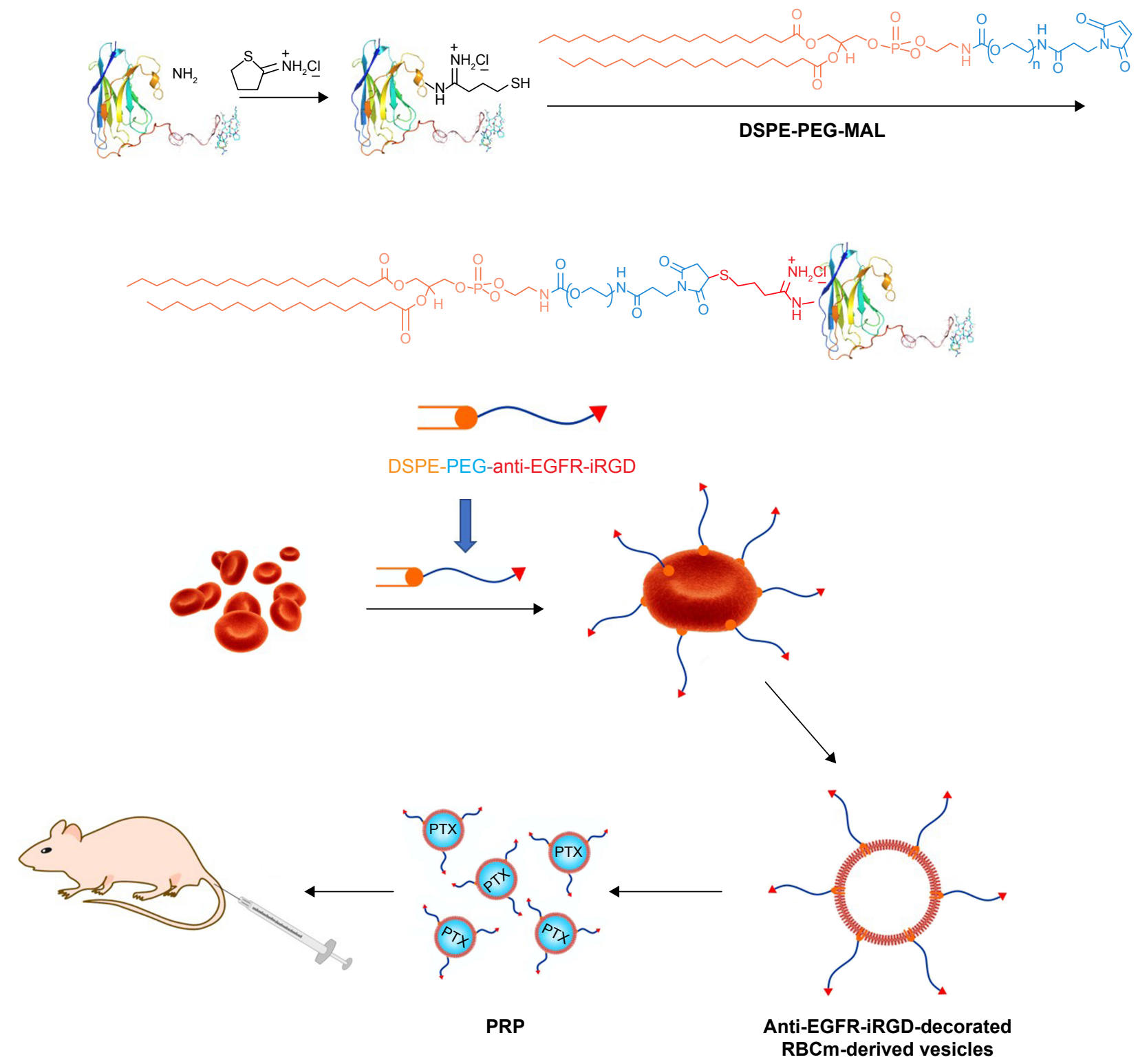

Figure I Schematic of the preparation process of PRP.

Notes: The process can be divided into the following three main steps: construction of DSPE-PEG-anti-EGFR-iRGD, collection and insertion of RBCm, and extrusion of anti-EGFR-iRGD-decorated RBCm and PTX. PRP, PTX-loaded RBCm-derived microvesicles functionalized with anti-EGFR-iRGD; RBCm-PTX, PTX-loaded RBCm-derived microvesicles.

Abbreviations: DSPE-PEG-MAL, I,2-distearoyl-sn-glycero-3-phosphoethanolamine-N-(maleimide[polyethylene glycol]-3400); PEG, poly(ethylene glycol); PTX, paclitaxel; $\mathrm{RBC}$, red blood cell; RBCm, RBC membrane.

\section{Animals}

Male BALB/c nude mice weighing 18-20 g (5-6 weeks old) were supplied by the Department of Experimental Animals, Yangzhou University (Yangzhou, People's Republic of China). The temperature and relative humidity were maintained at $25^{\circ} \mathrm{C}$ and $45 \%-55 \%$, respectively. All care and handling of the animals were performed in accordance with the guidelines approved by the Animal Care Committee at Nanjing Drum Tower Hospital (Nanjing, the People's Republic of China), and all the animal experiments conducted in this study were approved by the Animal Care Committee at Nanjing Drum Tower Hospital.

\section{Synthesis of anti-EGFR-iRGD and DSPE-PEG-anti-EGFR-iRGD}

Recombinant protein anti-EGFR-iRGD was prepared as previously reported. ${ }^{29,30}$ The anti-EGFR-iRGD was then used to prepare DSPE-PEG-anti-EGFR-iRGD, using a method previously reported. ${ }^{28,31}$ In brief, DSPE-PEG-MAL was mixed with anti-EGFR-iRGD at a 1:1 molar ratio 
(anti-EGFR-iRGD:DSPE-PEG-MAL=1:1) in Hepes ( $\mathrm{pH}=6.5$ ). The reaction mixture was gently stirred at room temperature for $24 \mathrm{~h}$, after which the resulting reaction mixture was placed in a dialysis bag (molecular weight cutoff $=7,000 \mathrm{Da}$ ) and dialyzed in PBS for $48 \mathrm{~h}$ to remove small molecules. The final solution in the dialysis bag was lyophilized and stored at $-20^{\circ} \mathrm{C}$ until used.

\section{Decoration of RBCs with DSPE-PEG- anti-EGFR-iRGD}

RBCs were extracted from fresh peripheral blood by centrifuging at $800 \times g$ for $5 \mathrm{~min}$ at $4^{\circ} \mathrm{C}$ and washed three times in cold saline $(0.9 \%$ normal saline $[\mathrm{NS}])$. After each centrifugation step, the supernatant was discarded. The washed RBCs were resuspended in $0.9 \% \mathrm{NS}$ and then incubated with DSPE-PEG-anti-EGFR-iRGD at $37^{\circ} \mathrm{C}$ for $30 \mathrm{~min}$ to form anti-EGFR-iRGD-inserted RBCs, following the methodology reported by Shi et al. ${ }^{32}$ The inserted RBCs were then washed three times with PBS before further use.

\section{Preparation of PTX-loaded $\mathrm{RBCm}$ nanosystem}

The RBCm-derived vesicles were prepared as described in a recent report. ${ }^{17}$ Briefly, the washed RBCs were lysed in $0.25 \times \mathrm{NS}$ and the ghosts were separated by collecting the pink pellet after centrifuging at $11,000 \mathrm{rpm}$ for $10 \mathrm{~min}$ at $4^{\circ} \mathrm{C}$. The RBC ghosts were subsequently extruded sequentially through 400 and $200 \mathrm{~nm}$ polycarbonate porous membranes using an Avanti Mini Extruder, yielding ghosts with the hydrodynamic diameter of $\sim 200 \mathrm{~nm}$. The mixture of fresh RBCm-derived vesicles and PTX was then extruded through $200 \mathrm{~nm}$ pores for at least 13 times, so that the mechanical force of the extruding process facilitated the encapsulation of PTX into the RBCm vesicles (RBCm-PTX). For the preparation of anti-EGFR-iRGD-RBCm-PTX (abbreviated to PRP), anti-EGFR-iRGD-inserted RBCs were used.

\section{Characterization of PTX-loaded $\mathrm{RBCm}$ nanosystem}

The particle sizes of PRP and RBCm-PTX were measured by dynamic light scattering (DLS; Malvern Zetasizer Nano ZS; Malvern Instruments, Malvern, UK) at $25^{\circ} \mathrm{C}$. To examine the long-term stability of the resulting nanosystem, they were suspended in 1× PBS for 2 weeks and their size and polydispersity index (PDI) were monitored by DLS.

The shape and surface morphology of RBCm-PTX and PRP were observed by transmission electron microscope (TEM). Uranyl acetate $(1 \%, w / v)$ was used to stain the membrane structure of the nanosystem. The color of the stained region appears as gray/white on the TEM images, as uranyl acetate is a negative staining reagent.

The drug loading efficiency was determined by highperformance liquid chromatography (HPLC), as previously described. ${ }^{33,34}$ PTX was quantified by ultraviolet detection at $227 \mathrm{~nm}$. The HPLC apparatus was calibrated using standard solutions of PTX dissolved in acetonitrile, $0.5-25 \mu \mathrm{g} / \mathrm{mL}$, with a correlation coefficient of $R^{2}=0.99$. The mobile phase $(1.0 \mathrm{~mL} / \mathrm{min})$ consisted of acetonitrile and water $(70: 30, \mathrm{v} / \mathrm{v})$. PTX was also dissolved in acetonitrile. The encapsulation efficiency was expressed as the percentage of PTX in the nanosystem relative to the initial amount of PTX used in their preparation.

To determine the in vitro release profile of PTX, several aliquots ( $1 \mathrm{~mL}$ each) of the nanosystem suspension were diluted to $5 \mathrm{~mL}$ with $\mathrm{PBS}(\mathrm{pH}=7.4)$, placed in sterile $50 \mathrm{~mL}$ Eppendorf tubes, incubated at $37^{\circ} \mathrm{C}$, and shaken horizontally at 160 strokes per minute. At predetermined time intervals, three sample tubes were withdrawn; the nanoparticles were separated by ultracentrifugation and then washed and prepared for the HPLC detection of PTX using the same procedure as described earlier.

Experimental values were the average of at least three different formulations.

\section{Fluorescent colocation}

DiO prestained PRP was used in this part. MNK45 cells $\left(3 \times 10^{5}\right.$ cells/well) were incubated in six-well plates (Corning Inc., Corning, NY, USA) containing culture medium at $37^{\circ} \mathrm{C}$ for $24 \mathrm{~h}$. Then, cell culture media containing anti-EGFRiRGD (labeled by Rhdamine B) decorated RBCm-PTX (RBCm labeled by DiO) (final anti-EGFR-iRGD concentration $150 \mathrm{ng} / \mathrm{mL}$ ) were added to each well, followed by incubation for another $4 \mathrm{~h}$ at $37^{\circ} \mathrm{C}$. After the medium was removed, the cells were washed with cold PBS, fixed with $4 \%$ paraformaldehyde in PBS, and then processed with DAPI stain for $10 \mathrm{~min}$. The cells were imaged using a fluorescence microscope.

\section{In vitro cytotoxicity assay}

MKN-45 cells were seeded $\left(8 \times 10^{3}\right.$ cells/well) in a 96-well plate (Corning Inc.) and incubated overnight. Free PTX, RBCm-PTX, PRP, and anti-EGFR-iRGD coadministration with RBCm-PTX were added to the cultures, respectively. After incubation for $24 \mathrm{~h}$, MTT solution was added and the cells were further incubated for $4 \mathrm{~h}$. Blank RBCmderived vesicles were also analyzed for in vitro cytotoxicity. 
The absorbance at $490 \mathrm{~nm}$ was measured using a BioTek ELx800 plate reader (BioTek Instruments, Inc., Winooski, VT, USA). The relative cell viabilities were then normalized to those of the control, which was set as $100 \%$.

\section{In vivo biodistribution imaging (near-infrared)}

A $0.1 \mathrm{~mL}$ suspension of MKN45 cells $\left(1 \times 10^{6}\right)$ was administrated via subcutaneous injection into the right armpits of male BALB/c nude mice to prepare the MKN45 tumorbearing nude mouse model. Once the tumor masses in the xenografts reached $300 \mathrm{~mm}^{3}, 200 \mu \mathrm{L}$ of physiological saline, DiR-prestained RBCm-PTX, PRP, or anti-EGFRiRGD coadministrated with DiR-prestained RBCm-PTX was intravenously (IV) administrated via the tail vein of the tumor-bearing nude mice at a dose of $10 \mathrm{mg} / \mathrm{kg}$ of PTX.

At predetermined time points, mice were anesthetized by isoflurane $(1.5 \%)$ and scanned using a Maestro ${ }^{\mathrm{TM}}$ Automated In Vivo Imaging System (Cambridge Research \& Instrumentation Inc., Woburn, MA, USA). The observation times were 2, 24, and $48 \mathrm{~h}$ after administration. For each near-infrared spectroscopy image, a corresponding X-ray image was taken to identify the anatomical location of the tumor.

\section{In vivo antitumor activity and safety study}

A tumor-bearing BALB/c nude mice model was prepared as before. When the tumor volume reached $\sim 100-200 \mathrm{~mm}^{3}$, the tumor-bearing mice were randomly assigned to five groups (each group contained at least five animals). These included a control group receiving IV injection physiological saline and four experimental groups receiving PTX, RBCm-PTX, PRP, and coadministered anti-EGFR-iRGD and RBCm-PTX, respectively. All experimental groups received PTX $10 \mathrm{mg} / \mathrm{kg}$, twice a week, for 2 weeks. Throughout the study, mice were weighed and tumors were measured with a caliper every 3 days. Tumor volumes were calculated using the following formula: $V=$ length $(\mathrm{mm}) \times$ width $^{2}\left(\mathrm{~mm}^{2}\right) \times 0.5$. Mice were sacrificed at the end of the experiment. Both tumor tissues and main organs (heart, liver, spleen, lung, and kidney) were dissected for histological observation. The tissues were fixed in $10 \%$ neutral buffered formalin, embedded in paraffin, sectioned at a thickness of $5 \mu \mathrm{m}$, stained with H\&E and then examined under optical microscopy.

\section{Statistical analysis}

All data were shown as the mean \pm SD. The SPSS 21.0 software was used for statistical analysis, and $t$-test and one-way analysis of variance were used for comparisons. $P<0.05$ was considered to indicate a statistically significant difference.

\section{Results}

\section{Decoration of RBCs with} anti-EGFR-iRGD

First, we decorated RBCs in vitro using anti-EGFR-iRGD. To simplify the preparation process, we used the protocol described by Shi et al. ${ }^{32}$ Anti-EGFR-iRGD was coupled with DSPE-PEG-MAL via Michael addition. The successful synthesis of DSPE-PEG-anti-EGFR-iRGD was tested by sodium dodecyl sulfate polyacrylamide gel electrophoresis (SDS-PAGE) analysis, as shown in Figure 2A. As shown in Figure 2A (Line 4), protein markers, purified anti-EGFRiRGD, the reaction product of the Michael addition, and DSPE-PEG-MAL were loaded in Lines 1-4, respectively. DSPE-PEG-MAL alone did not result in any band on the gel. Anti-EGFR-iRGD, which has a size of $18 \mathrm{kDa}$, showed a band corresponding to a molecular weight between 26 and $17 \mathrm{kDa}$ (Figure 2A, Line 2). A successful conjugation of anti-EGFR-iRGD and DSPE-PEG-MAL was confirmed by the band observed above, the anti-EGFR-iRGD band (Figure 2A, Line 3), as DSPE-PEG-anti-EGFR-iRGD did not migrate as fast as the free anti-EGFR-iRGD molecules under electrophoresis condition. Bands corresponding to anti-EGFR-iRGD could be seen in both Lines 3 and 2, we speculated that some free anti-EGFR-iRGD remained in the product solution after the reaction of anti-EGFR-iRGD and DSPE-PEG-MAL, indicating that the reaction efficiency of anti-EGFR-iRGD and DSPE-PEG-MAL was not $100 \%$.

The washed human RBCs were then incubated with DSPE-PEG-anti-EGFR-iRGD for $30 \mathrm{~min}$ at $37^{\circ} \mathrm{C}$. The incorporation of anti-EGFR-iRGD molecules into RBCms was observed by flow cytometry, using the anti- $6 \times$ His tag antibody. As an increasing amount of DSPE-PEG-anti-EGFRiRGD was fed into the incubation system, the percentage of RBCs with a detectable Dylight ${ }^{\circledR} 650$ signal increased, as shown in Figure $2 \mathrm{~B}$ and C. For $10^{6} \mathrm{RBCs}$, the proportion of positive RBCs reached a plateau when $>100 \mathrm{ng}$ of DSPEPEG-anti-EGFR-iRGD was added (Figure 2B). Nonlipid anti-EGFR-iRGD was also incubated with the same amount of RBCs to determine whether nonlipid anti-EGFR-iRGD could bind to suspended RBCs. As shown in Figure 2D, no significant binding of nonlipid anti-EGFR-iRGD to RBCs was observed. These results indicated that free anti-EGFRiRGD could not bind to RBCs, whereas after reaction with DSPE-PEG-MAL, anti-EGFR-iRGD was successfully inserted into RBCs. 
A

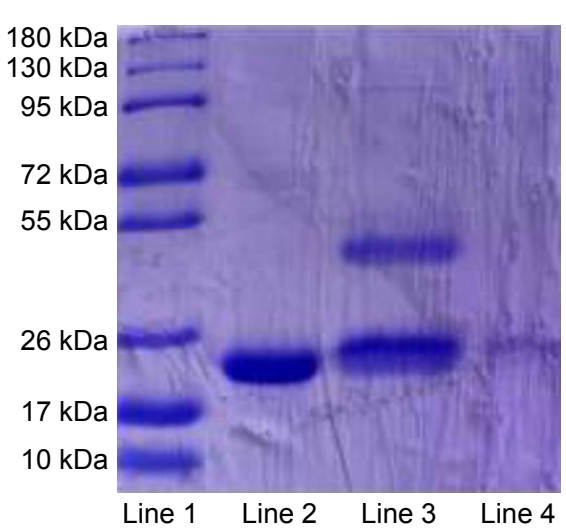

B

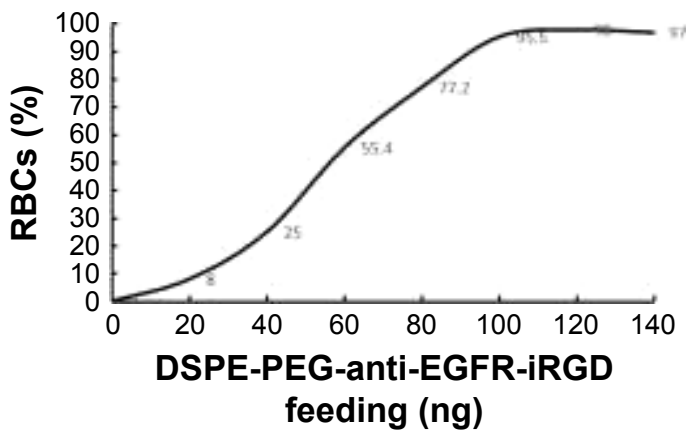

\section{C}
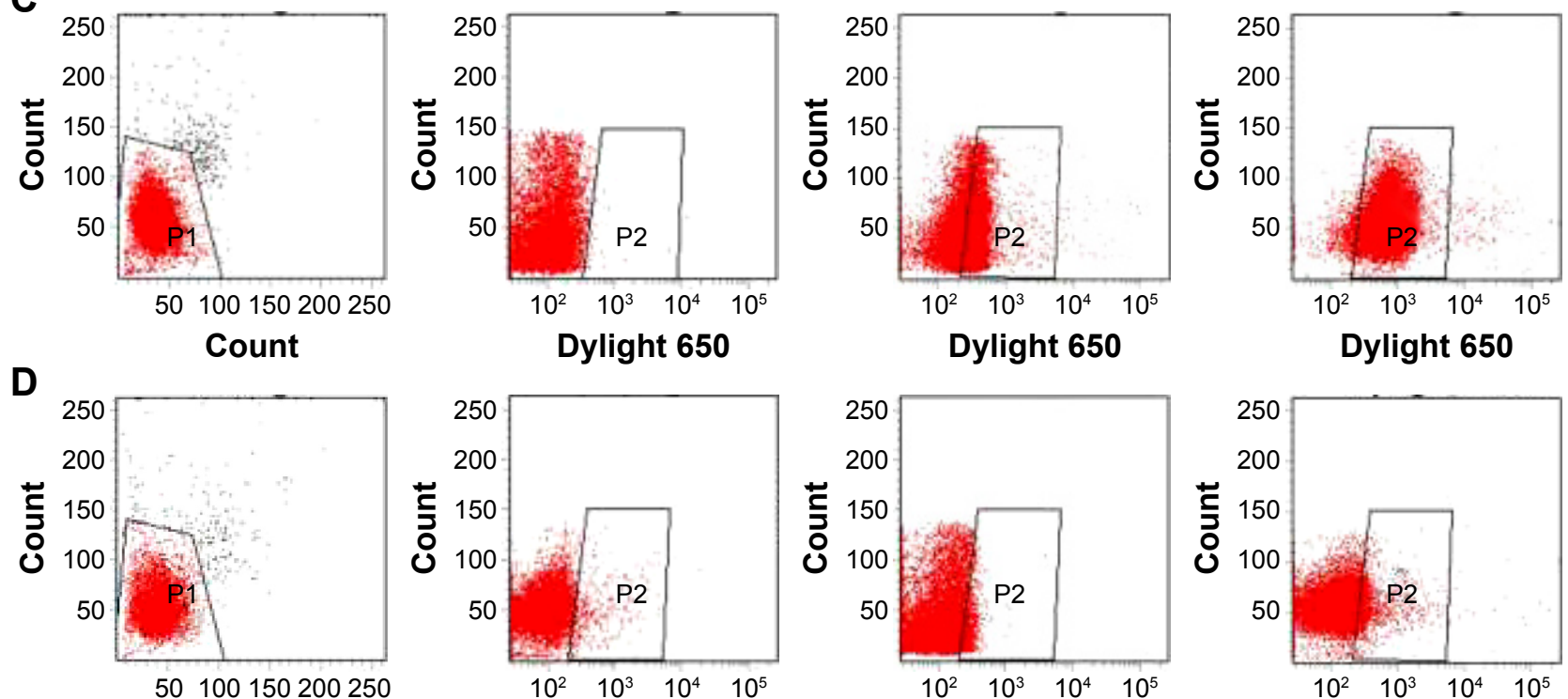

Count
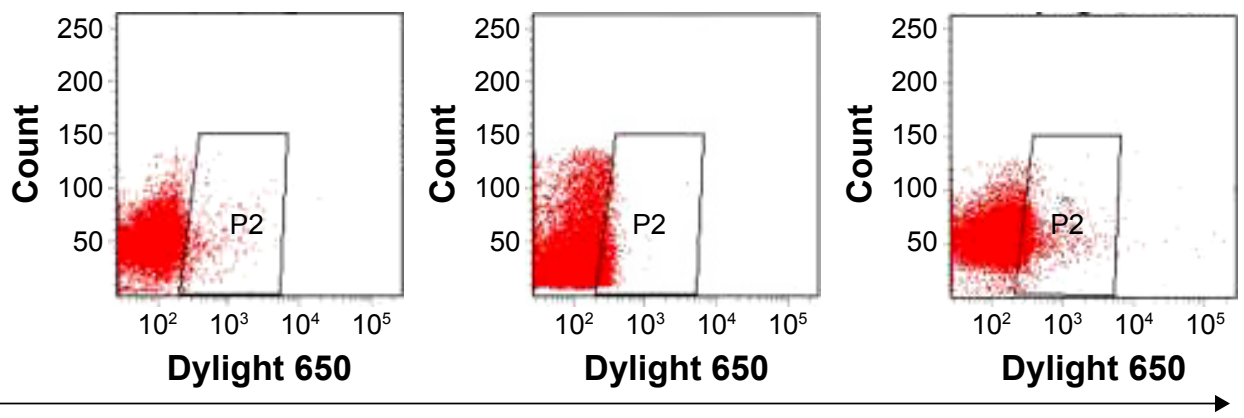

Figure 2 Decoration of RBCs with anti-EGFR-iRGD.

Notes: (A) Yield of DSPE-PEG-anti-EGFR-iRGD: Line I, prestained color protein marker; line 2, anti-EGFR-iRGD; line 3, reaction product of DSPE-PEG-MAL and antiEGFR-iRGD after filtration using an ultrafiltration tube; line 4, DSPE-PEG-MAL. (B) Flow cytometry results of $10^{6}$ RBCs incubated with different amounts of DSPE-PEG-antiEGFR-iRGD. (C) A total of $10^{6}$ RBCs incubated with different amounts of anti-EGFR-iRGD (Dylight ${ }^{\circledR} 650$, from left to right: 10 , 60, and I00 ng). (D) Flow cytometry results of $10^{6}$ RBCs incubated with different amounts of anti-EGFR-iRGD (Dylight ${ }^{\circledR}$ 650, from left to right: 10, 60, and I00 ng). DSPE-PEG-anti-EGFR-iRGD, reaction product of DSPE-PEG-MAL and anti-EGFR-iRGD.

Abbreviations: DSPE-PEG-MAL, I,2-distearoyl-sn-glycero-3-phosphoethanolamine-N-(maleimide[polyethylene glycol]-3400); PEG, poly(ethylene glycol); PTX, paclitaxel; RBCs, red blood cells.

\section{Preparation and characterization of PRP and RBCm-PTX}

Once anti-EGFR-iRGD had been successfully inserted into RBCs, the decorated RBCs were subjected to obtain $\mathrm{RBCm}$ ghosts as previously reported. ${ }^{17}$ These $\mathrm{RBCm}$ ghosts were then used to fabricate RBCm-PTX and PRP using an Avanti Mini Extruder. The particle size of RBCm-PTX and PRP was measured by DLS. The average particle size of RBCm-PTX was $168.7 \pm 2.2 \mathrm{~nm}$, with a PDI of $0.200 \pm 0.002$. For PRP, the average particle size was $\sim 171.7 \pm 4.7 \mathrm{~nm}$, with a PDI of $0.214 \pm 0.005$. The encapsulation efficiency and the drug-loading efficiency of PRP were $60.00 \%$ and $34.07 \%$, respectively. TEM was used to observe the morphology of the nanosystem. Figure $3 \mathrm{~A}$ and $\mathrm{B}$ shows the representative TEM images of RBCm-PTX and PRP, respectively. Figure $3 \mathrm{C}$ shows RBCm-PTX stained by uranyl acetate, the particle size observed was in good agreement with the DLS measurements. The gray/white region on the TEM image (Figure 3C) represents the RBCm, which was $\sim 7-8 \mathrm{~nm}$ in thickness, consistent with a previous study. ${ }^{12}$

To investigate the stability of the nanosystems, RBCmPTX and PRP were suspended in PBS and then monitored 
A

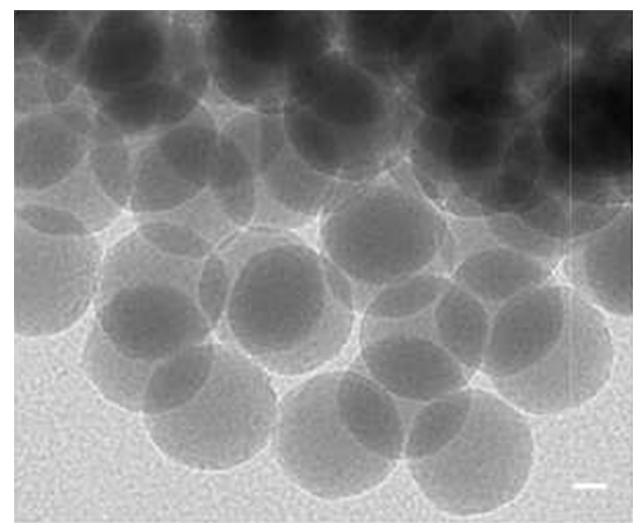

B

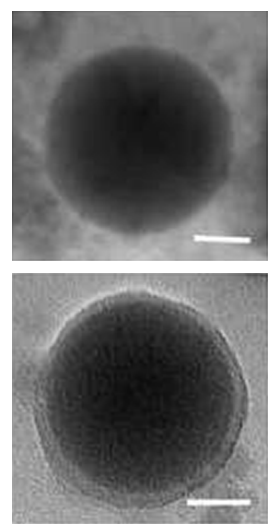

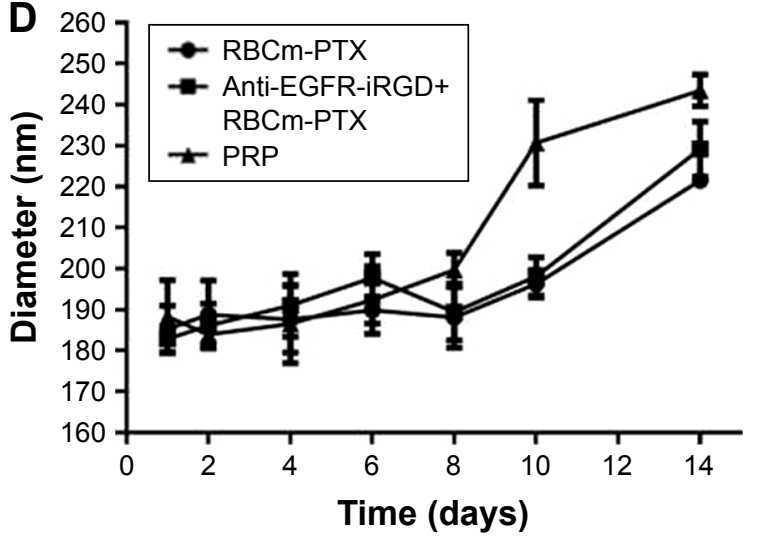

$\mathbf{E}$

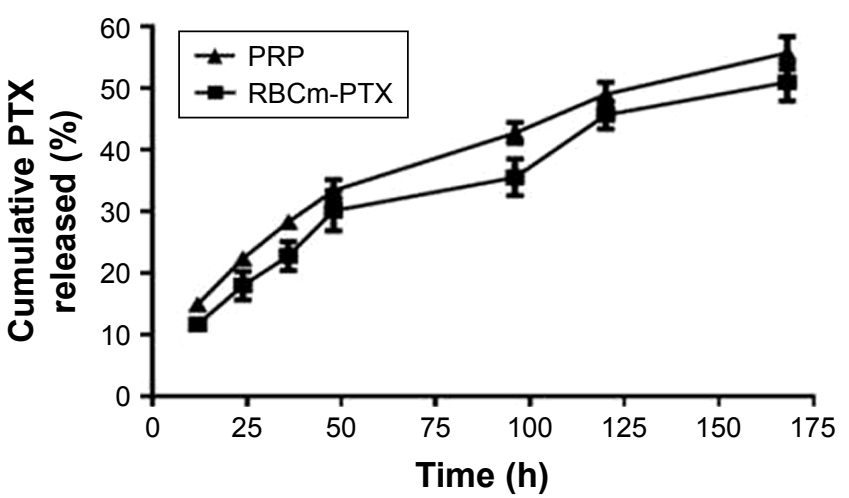

Figure 3 Characterization of PRP and RBCm-PTX.

Notes: (A) Representative TEM images of RBCm-PTX, scale bars $=50 \mathrm{~nm}$. (B) Representative TEM image of PRP, scale bars $=50 \mathrm{~nm}$. (C) RBCm-PTX sample was negatively stained with uranyl acetate and subjected for TEM imaging, scale bars $=50 \mathrm{~nm}$. (D) Stability of PRP and RBCm-PTX in PBS. (E) In vitro release profile of PRP and RBCm-PTX in PBS. PRP, PTX loaded RBCm-derived microvesicles functionalized with anti-EGFR-iRGD.

Abbreviations: PTX, paclitaxel; RBCs, red blood cells; RBCm, RBC membrane; TEM, transmission electron microscope.

by DLS for 2 weeks (Figure 3D). The particle size of RBCm-PTX increased from $\sim 165$ to $222 \mathrm{~nm}$ and that of PRP increased from $\sim 164$ to $270 \mathrm{~nm}$, while the PDI remained relatively unchanged at 0.21 . These results indicated that PRP and RBCm-PTX remained relatively stable in PBS for about 8 days and the particle size gradually increased. Moreover, the incorporation of anti-EGFR-iRGD into RBCm-PTX did not significantly affect the stability of RBCm-PTX.

The in vitro release behavior of PTX from RBCm-PTX and PRP is illustrated in Figure 3E. As can be observed, during the first $48 \mathrm{~h}$, PTX release was rapid, with $\sim 30.12 \% \pm 3.26 \%$ released from RBCm-PTX and $33.39 \% \pm 1.76 \%$ from PRP. During the second $48 \mathrm{~h}$ and the subsequent 3 days, release of PTX continued at a slower rate and $~ 50.94 \% \pm 3.00 \%$ from $\mathrm{RBCm}-\mathrm{PTX}$ and $55.65 \% \pm 2.64 \%$ from PRP were released. This indicated that the release behavior of PTX from these nanosystems followed a biphasic pattern.

To visualize the successful incorporation of DSPE-PEGanti-EGFR-iRGD into RBCm-PTX, PRP was washed by PBS for three times to remove free anti-EGFR-iRGD and the washed
PRP was added into culture system of MKN45 in a six-well plate. After $4 \mathrm{~h}$ incubation, cells were washed and observed under fluorescence microscopy. As shown in Figure 4, both RBCm (green) and anti-EGFR-iRGD (red) could be observed around the nucleus (DAPI) in the resulting images, indicating the colocation of RBCm and anti-EGFR-iRGD.

\section{In vitro cytotoxicity assay}

For the cytotoxicity assays, MKN45 cells were treated with different concentrations of PTX, RBCm-PTX, PRP, and anti-EGFR-iRGD coadministrated with RBCm-PTX for $24 \mathrm{~h}$. Blank RBCm-derived vesicles were also tested for their cytotoxicity. Results showed that RBCm-derived vesicles had no significant effect on the cell vitality of MKN45 for any of the preset concentrations (data not shown). As shown in Figure 5, the IC50 of PTX was $5.1 \mathrm{ng} / \mathrm{mL}$, with no significant difference in cell viability between groups at various drug concentrations. Based on these results, we can infer that PTX does not lose its cytotoxicity toward MKN45 cells as a result of being loaded into $\mathrm{RBCm}$-derived vesicles. 

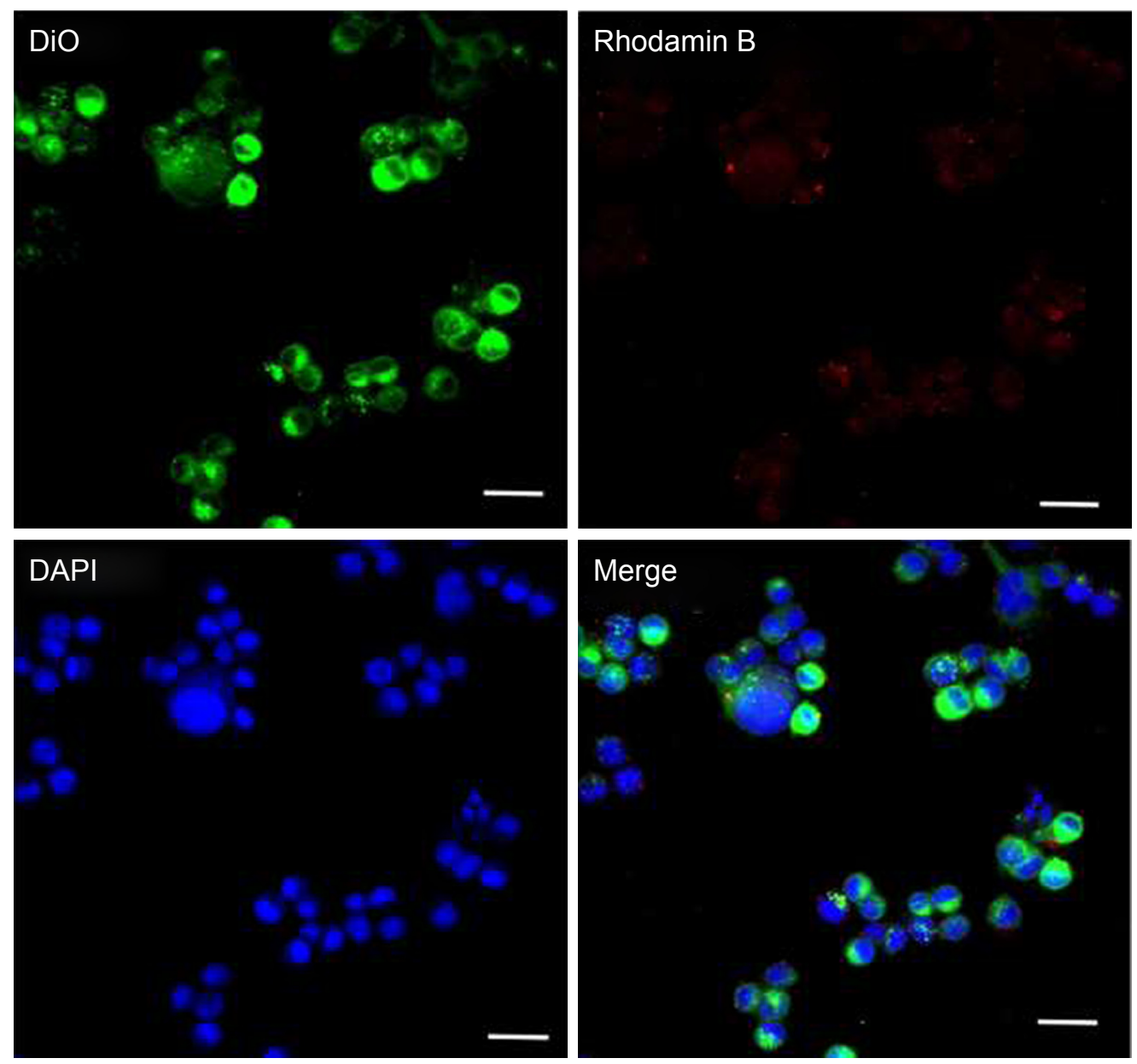

Figure 4 Fluorescent colocation of PRP with MKN45 cells.

Notes: Representative immunofluorescence images of MKN45 cells, which were stained with DAPI (blue). Anti-EGFR-iRGD was prestained with Rhodamine B (red), RBCm-PTX was prestained with DiO (green), scale bar $=20 \mu \mathrm{m}$. PRP, PTX-loaded RBCm-derived microvesicles functionalized with anti-EGFR-iRGD.

Abbreviations: DiO, 3,3'-dioctadecyloxacarbocyanine perchlorate; PTX, paclitaxel; RBC, red blood cell; RBCm, RBC membrane.

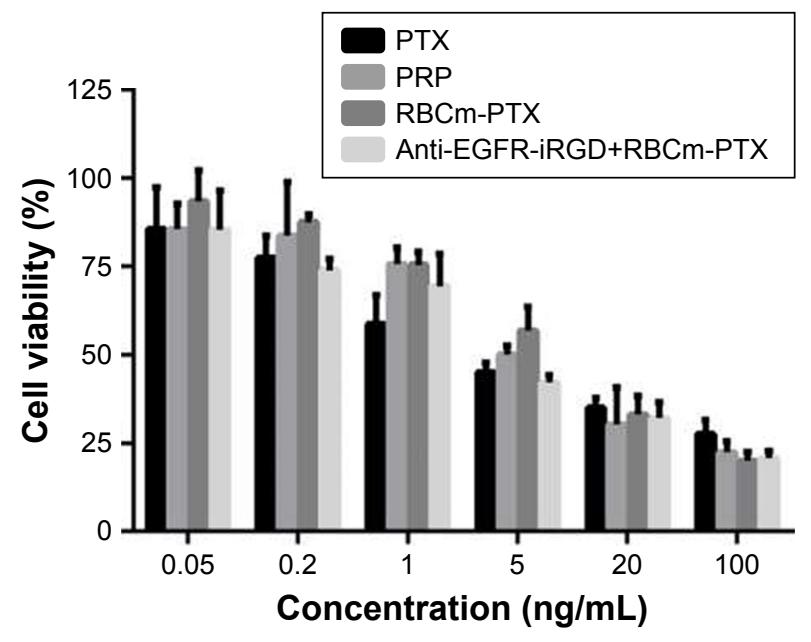

Figure 5 In vitro cytotoxicity assay.

Notes: In vitro cytotoxicity of PTX, RBCm-PTX, PRP, and coadministration of anti-EGFR-iRGD and RBCm-PTX after incubation for $24 \mathrm{~h}$. Error bars indicate $\mathrm{SD}(\mathrm{n}=3)$. PRP, PTX-loaded RBCm-derived microvesicles functionalized with antiEGFR-iRGD.

Abbreviations: PTX, paclitaxel; RBC, red blood cell; RBCm, RBC membrane.

\section{In vivo tumor targeting and tissue distribution}

The in vivo tumor accumulation and tissue distribution of these nanosystem were examined by a Maestro ${ }^{\mathrm{TM}}$ Automated In Vivo Imaging System, using fluorescein DiR. As shown in Figure 6A, PRP accumulated in the tumorous lesion within $2 \mathrm{~h}$ of administration. After this time, the PRP group showed markedly enhanced fluorescence at the tumor site, whereas the anti-EGFR-iRGD coadministrated with RBCm-PTX group and the RBCm-PTX group showed no obviously accumulated fluorescence, although the signal of anti-EGFRiRGD coadministrated with RBCm-PTX group appeared to be slightly stronger than that of the RBCm-PTX group. At $48 \mathrm{~h}$ after drug injection, enhanced fluorescence could still be detected in the PRP group and the fluorescence signal of DiR in the tumor site was significantly stronger than that of the RBCm-PTX group, whereas the anti-EGFR-iRGD 


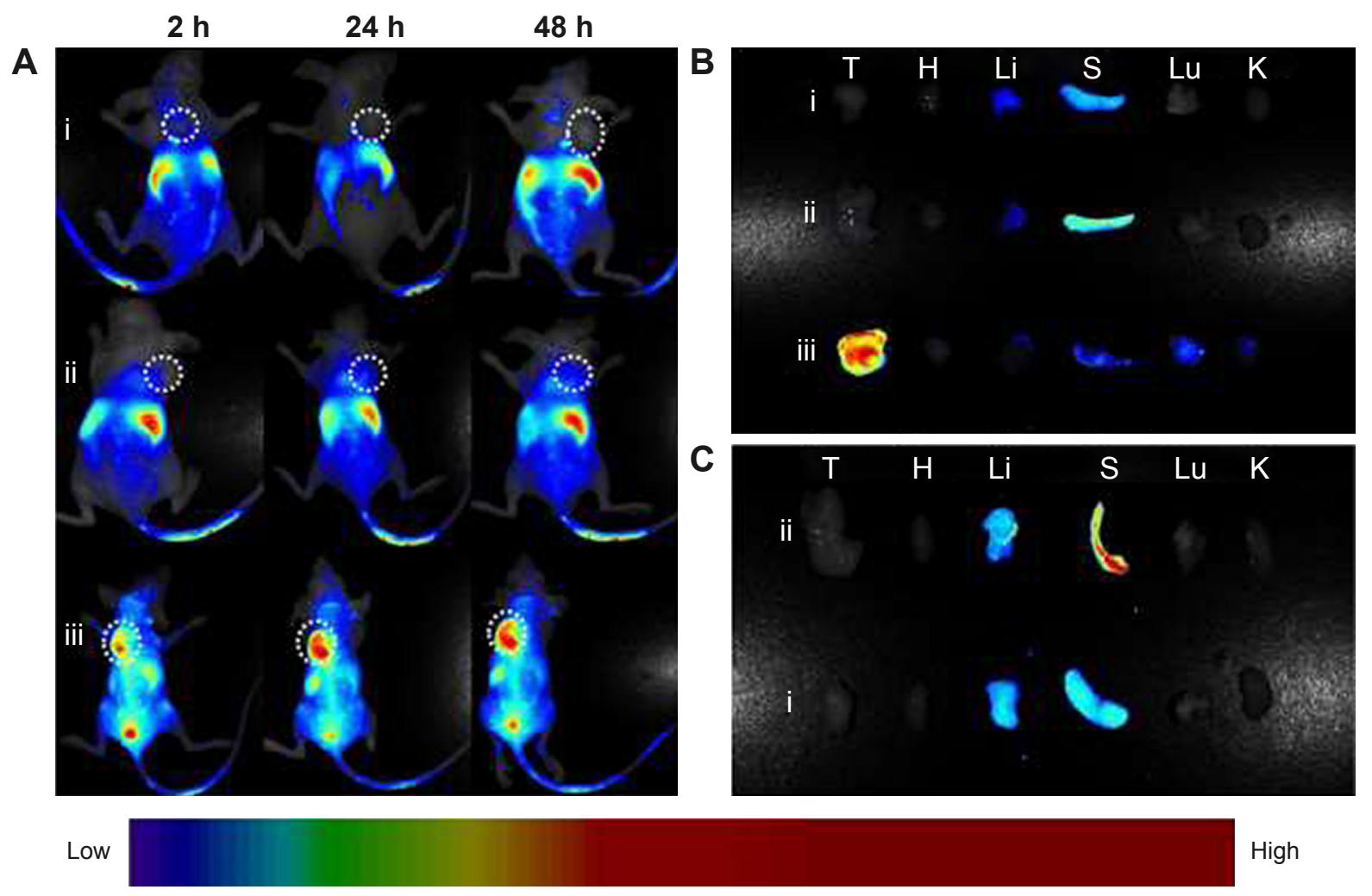

Figure 6 In vivo targeting ability of PRP.

Notes: (A) In vivo fluorescence imaging of the MKN45 tumor-bearing nude mice at 2, 24, and $48 \mathrm{~h}$ after intravenous injection of RBCm-PTX (i), coadministration of antiEGFR-iRGD and RBCm-PTX (ii) and PRP (iii), and all the erythrocyte membranes used here were labeled by DiR, at dose of $20 \mathrm{nmol} / \mathrm{kg}$. The white colored dotted circles indicated the tumor sites. (B) Ex vivo fluorescence imaging of the excised tumors and major organs at $48 \mathrm{~h}$ postinjection of all three groups. (C) Ex vivo fluorescence imaging of the excised tumors and major organs at $48 \mathrm{~h}$ postinjection of RBCm-PTX-treated group (i) and RBCm-PTX coadministration with anti-EGFR-iRGD-treated group (ii). PRP, PTX-loaded RBCm-derived microvesicles functionalized with anti-EGFR-iRGD.

Abbreviations: DiR, I,I'-dioctadecyltetramethyl indotricarbocyanine iodide; H, heart; K, kidney; Lu, lung; Li, liver; PTX, paclitaxel; RBC, red blood cell; RBCm, RBC membrane; S, spleen; T, tumor.

coadministrated with RBCm-PTX group showed no detectable enhancement of fluorescence at the tumor site.

To observe the fluorescence more clearly, the tumor-bearing animals were sacrificed $48 \mathrm{~h}$ postinjection and tumors and major organs were excised and collected. As shown in Figure 6B and $\mathrm{C}$, the tumor of PRP group gave the strongest fluorescence signals among all three groups (Figure 6B), whereas the tumors of the other two groups had similar fluorescence intensity (Figure 6C). This indicates that PRP has enhanced tumor-targeting ability compared with RBCm-PTX and coadministration of anti-EGFR-iRGD and RBCm-PTX.

\section{In vivo antitumor study}

We next sought to determine whether anti-EGFR-iRGD decoration could improve the therapeutic efficiency of RBCm-PTX. A mouse model subcutaneously bearing MKN45 tumor was established. MKN45-bearing mice were treated with different formulations of PTX twice a week for 2 weeks, by caudal vein injection. Mice treated with PBS served as a control group. Figure 7A shows the growth curves of subcutaneous tumors. The MKN45 tumors in the control group grew rapidly, increasing about eightfold on average by day 15, while those treated with PTX and PTX-loaded $\mathrm{RBCm}$ vesicles showed reduced growth to various extents. At the end point, the tumor sizes $\left(\mathrm{mm}^{3}\right.$, mean $\left.\pm \mathrm{SD}, \mathrm{n}=5\right)$ for each group were $1,006 \pm 145$ for the control group, $793 \pm 73$ for the PTX group, $673 \pm 52$ for the RBCm-PTX group, $607 \pm 66$ for the anti-EGFR-iRGD coadministrated with RBCm-PTX group, and $389 \pm 72$ for the PRP group. In the PTX, RBCmPTX, anti-EGFR-iRGD coadministrated with RBCm-PTX, and PRP groups, tumor volumes were reduced by $\sim 21,33,40$, and $61 \%$, respectively, relative to the control group. Tumors were weighted immediately after harvesting; the weights (g, mean $\pm \mathrm{SD}, \mathrm{n}=5$ ) were $0.78 \pm 0.24$ for the control group, $0.46 \pm 0.17$ for the PTX group, $0.33 \pm 0.09$ for the RBCmPTX, $0.24 \pm 0.05$ for the anti-EGFR-iRGD coadministrated with RBCm-PTX group, and $0.13 \pm 0.07$ for the PRP group, as shown in Figure 7B. By contrast, the RBCm-PTX and anti-EGFR-iRGD coadministrated with RBCm-PTX group showed higher tumor inhibition rates than the PTX group. 
The PRP group showed enhanced therapeutic efficacy compared with the other groups. Combining these findings with the in vivo distribution results, we speculated that antiEGFR-iRGD decoration could enhance tumor targeting and improve therapeutic efficiency of RBCm-PTX.

Furthermore, mice tolerated the treatment very well, with no change in motility or eating habits. As shown in Figure 7C, none of the mice treated with PRP showed obvious body weight loss during the treatment, in contrast to mice treated with combined anti-EGFR-iRGD and RBCm-PTX, RBCmPTX, or PTX alone. The differences in body weight between the PRP group and other groups were significant $(P<0.05)$. As shown in Figure 7D, no abnormalities were observed in the H\&E-stained sections of the main organs.

\section{Discussion}

In this study, we designed and manufactured a drug delivery system, named PRP. In this system, PTX was loaded into erythrocyte membrane-derived microvesicles and a fusion

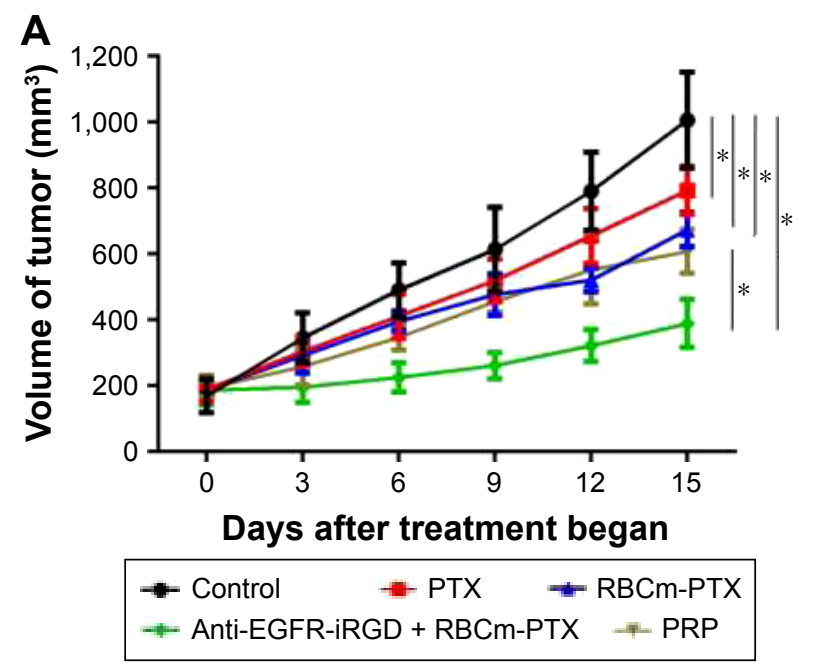

protein (anti-EGFR-iRGD) was used to decorate erythrocyte membrane for tumor targeting. GC cell line, MKN45, which has been reported to express $\mathrm{iRGD}^{35}$ receptors and was confirmed to be EGFR positive in our laboratory, was chosen as a model tumor cell in vitro and in vivo. We investigated for the first time, the therapeutic efficacy of PRP in the GC cell line and in tumor-bearing mice. Both anti-EGFR-iRGD decorated and undecorated RBCm-PTX had uniformly spherical shape and size, a relatively narrow distribution, similar stability, and PTX release behavior in vitro. The targeting ability of the PRP in MKN45 cells, compared to anti-EGFRiRGD coadministrated with RBCm-PTX, RBCm-PTX, and PTX, was enhanced both in vitro and in vivo, and PRP had improved therapeutic efficiency.

The results presented here indicate that PRP performed better than nonmodified RBCm-PTX or PTX alone. The procedure for constructing this tumor-targeting PTX delivery system is relatively straightforward and does not require organic solvents. We used an Avanti Mini Extruder to extrude

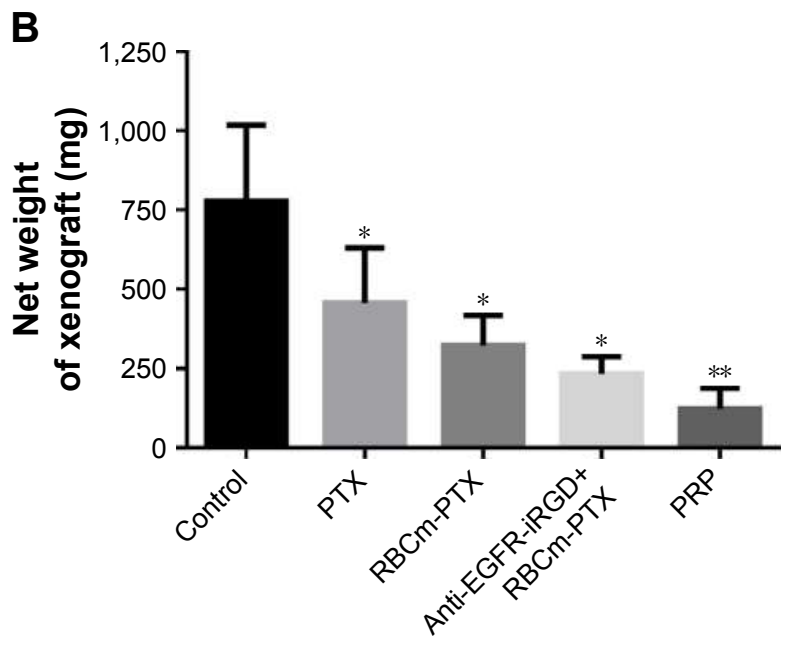

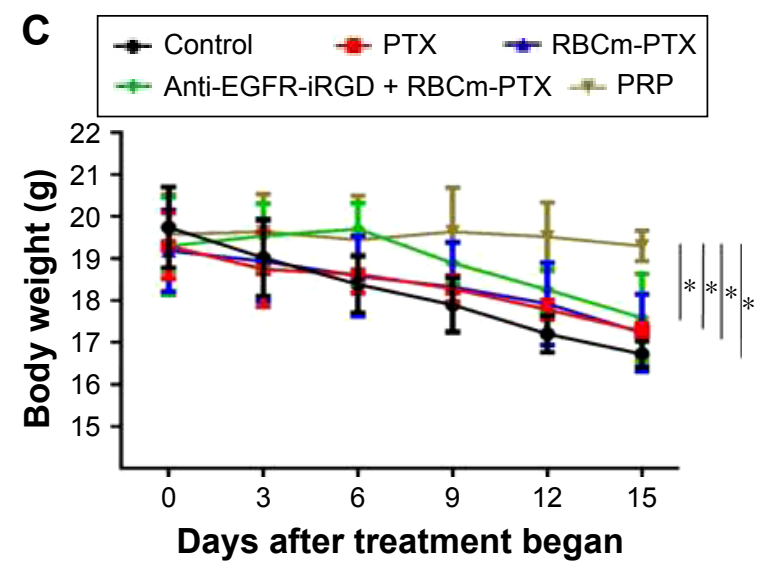

Figure 7 (Continued) 
D
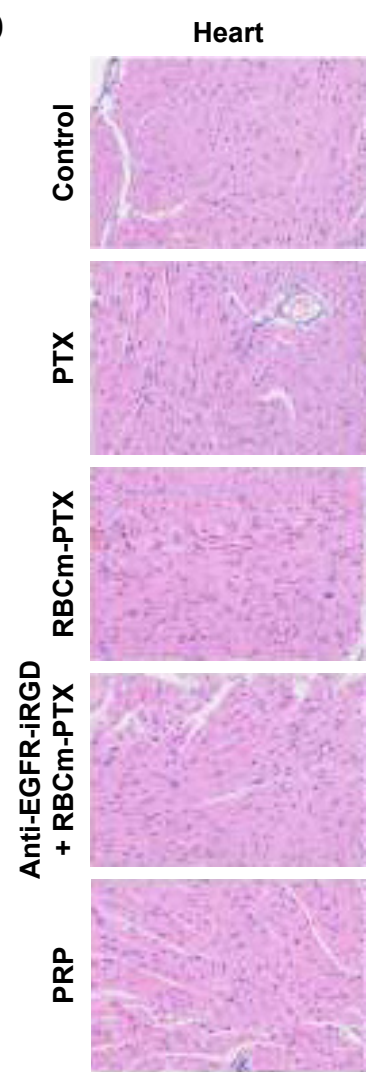

Liver
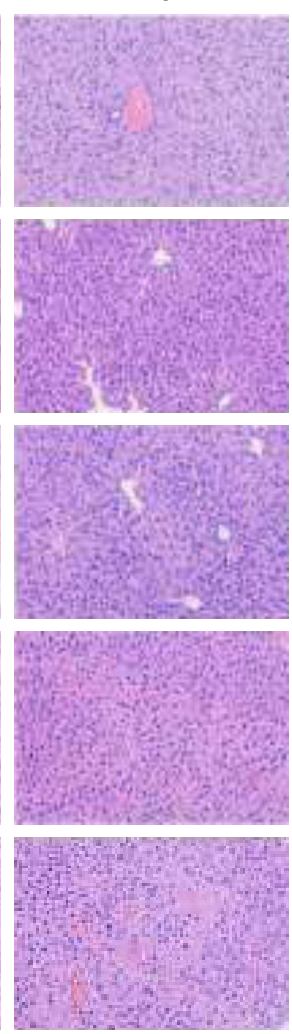

Spleen
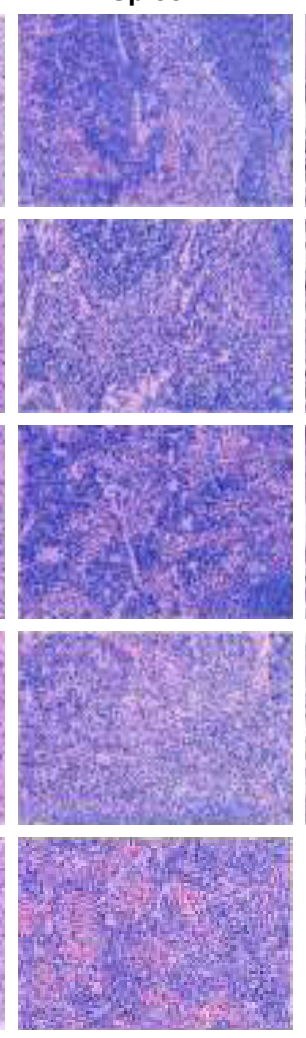
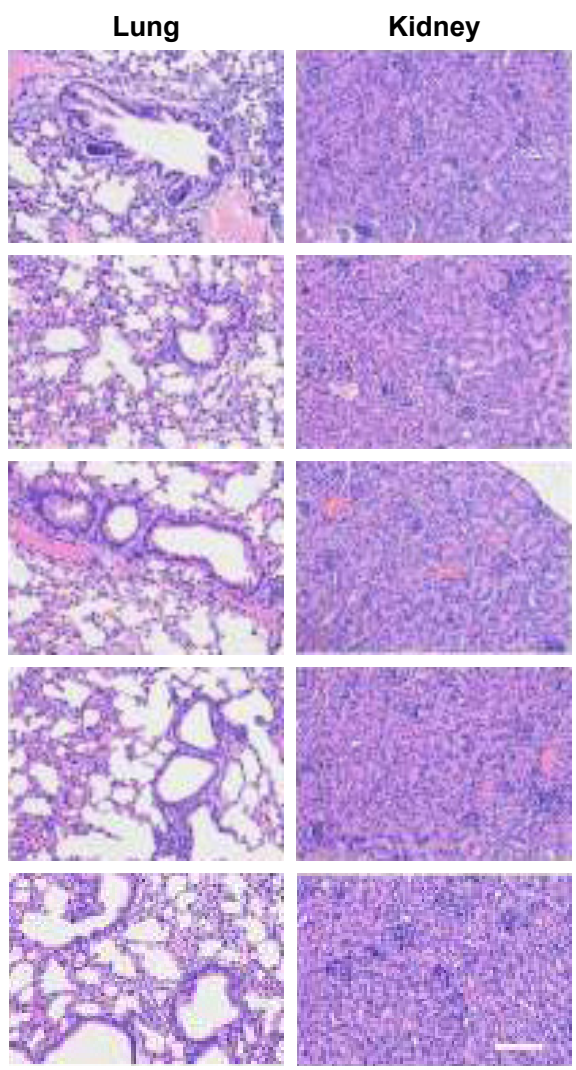

Figure 7 In vivo antitumor activity and safety study.

Notes: Mice-bearing MKN45 subcutaneously were used as an animal model. Mice were treated by tail vein injection of PBS, PTX alone, RBCm-PTX, coadministration of anti-EGFR-iRGD and RBCm-PTX, or PRP twice a week for 2 weeks. (A) The tumor growth curves, (B) the net weight of xenografts after harvesting, and (C) the body weight change of the tumor-bearing mice. Data are represented as mean \pm SEM $(n=5)$. One-way analysis of variance was used for the analysis of tumor volume and body weight. $* P<0.05$ and $* * P<0.00 I$. (D) Main organs were dissected for $\mathrm{H} \& \mathrm{E}$ staining on the 15 th day after treatment. Main organs, including heart, liver, spleen, lung, and kidney. Inflammatory cells' infiltration could be seen in some cases, while none significant abnormal damage was observed. Scale bar=I00 $\mu$ m. PRP, PTX-loaded RBCm-derived microvesicles functionalized with anti-EGFR-iRGD.

Abbreviations: PTX, paclitaxel; RBC, red blood cell; RBCm, RBC membrane; SEM, standard error of the mean.

the RBC ghosts, which gave the erythrocyte membranederived microvesicles their relatively uniform size and shape. In clinical settings, Abraxane ${ }^{\circledR}$ is widely used to treat patients with advanced cancers; the production of Abraxane ${ }^{\circledR}$ involves emulsification $^{36}$ and high-pressure homogenous, both of which are more complicated than the methods we used in this research. Various strategies have previously been reported for fabricating PTX-loaded nanoparticles. ${ }^{37-43}$ For instance, biodegradable polymeric nanoparticles such as poly(lacticco-glycolic acid) (PLGA) are often used as vectors; however, organic solvent such as dichloromethane, acetone are needed for the preparation of the PLGA polymeric cores. ${ }^{12,44}$ Even small residual amounts of dichloromethane have been shown to be toxic to multiple vital organs, including the liver, kidney, lungs, and neural system. ${ }^{45}$ By contrast, our procedure is free of organic solvent and is relatively safe with respect to both manufacture and clinical use.
As well as the use of natural materials, our system required less artificial modification compared with others. RBCs are among the most commonly used and extensively studied natural carriers, with applications involving many different medicines and chemicals. ${ }^{46-48}$ With the development of nanotechnology, biomembranes have also been comprehensively studied. ${ }^{48,49} \mathrm{RBCs}$ are the most robust source for biomembranes, as they have a long duration of circulation in the blood, will not activate the immune system in vivo, and can be sustainably produced by and quantitatively isolated from the human body. Owing to these properties, they have attracted extensive interest from researchers to aiming to design RBC-mimicking and $\mathrm{RBCs} / \mathrm{RBCm}$-based drug delivery systems. ${ }^{50}$ In the present work, membranes were collected from RBCs by hypotonic treatment and washed with physiological saline. This processing method has been tested by many other researchers in the past. ${ }^{12,13,15}$ 
In our previous work, we constructed and expressed a fusion protein consisting of an EGFR single-domain antibody and iRGD peptide, named anti-EGFR-iRGD. We showed that anti-EGFR-iRGD could improve the permeability and efficacy of anticancer drugs such as doxorubicin, bevacizumab, and nanoparticles in multicellular spheroids of $\mathrm{GC}^{29}$ and enhance drug permeability to both two-dimensional cells and threedimensional multicellular spheroids. In order to improve the targeting and penetration ability of cell membrane-based nanocarriers, linker-based techniques were used to functionalize these nanocarriers with anti-EGFR-iRGD. In 2013 and 2014, a lipid insertion technique using DSPE-PEG-MAL was successively reported by two independent research groups as a robust and simple way to functionalize RBCms. ${ }^{28,32}$ This method was used in the present research, in which it was also proved to be effective. Later in 2016, Zhou et $\mathrm{al}^{51}$ reported using succinimidyl-([N-maleimidopropionamido]-polyethylene glycol) ester (NHS-PEG-MAL), a bifunctional linker, to stably conjugate bioactive molecules such as proteins on biomembranes. After functionalization, both the desired functional molecules and the cell membrane maintained their original properties and may also have presented some improved features in the study sample. ${ }^{51}$ This facile approach makes it possible to expand the application of these platform strategies to the membrane of a variety of cells and the corresponding derivative-coating nanoparticles and/or vesicles. Potential cell types that could be used include leukocytes, thrombocytes, cancer cells, and stem cells; more biofunctional molecules could be designed in the future to guide or target therapies for dozens of diseases. We are now focusing on targeting biomembrane-derived nanoparticles for tumor immunotherapy.

\section{Conclusion}

We designed and successfully prepared a novel nanosystem based on anti-EGFR-iRGD-decorated erythrocyte membrane for the targeted delivery of PTX to GC. This system named PRP, enabled enhanced accumulation of the drug in tumors, and improved tumor suppression in GC. The present simple strategy pioneers the idea of translating nanotechnologymodified traditional medicines from bench to bedside. Our team is currently carrying out related researches, including the development of targeted biomembrane-derived nanoparticles for tumor immunotherapy.

\section{Acknowledgments}

The authors gratefully acknowledge the generous financial support from the National Key Research and Development Program of China (number 2017YFC1308900) and
National Natural Science Foundation of China (grant numbers $81502037,81672367,81672398,81220108023$, and $81572601)$. The abstract of this article was presented at the Society for Immunotherapy of Cancer as a poster presentation with interim findings and also presented at the America Society of Clinic Oncology as a poster presentation with interim findings. The poster's abstract was published in Journal of Clinical Oncology: http://ascopubs.org/doi/abs/10.1200/ JCO.2017.35.15 suppl.e14047.

\section{Disclosure}

The authors report no conflicts of interest in this work.

\section{References}

1. Chen W, Zheng R, Baade PD, et al. Cancer statistics in China, 2015. CA Cancer J Clin. 2016;66(2):115-132.

2. Hamada H, Ishihara K, Masuoka N, Mikuni K, Nakajima N. Enhancement of water-solubility and bioactivity of paclitaxel using modified cyclodextrins. J Biosci Bioeng. 2006;102(4):369-371.

3. Mathew AE, Mejillano MR, Nath JP, Himes RH, Stella VJ. Synthesis and evaluation of some water-soluble prodrugs and derivatives of taxol with antitumor-activity. J Med Chem. 1992;35(1):145-151.

4. Rowinsky EK. Clinical pharmacology of taxol. J Natl Cancer Inst Monogr. 1993;(15):25-37.

5. Hajek R, Vorlicek J, Slavik M. Paclitaxel (Taxol): a review of its antitumor activity in clinical studies minireview. Neoplasma. 1996;43: $141-154$.

6. Rowinsky EK, Eisenhauer EA, Chaudhry V, Arbuck SG, Donehower RC. Clinical toxicities encountered with paclitaxel (Taxol). Semin Oncol. 1993;20(4 Suppl 3):1-15.

7. Copp JA, Fang RH, Luk BT, et al. Clearance of pathological antibodies using biomimetic nanoparticles. Proc Natl Acad Sci USA. 2014;111(37): 13481-13486.

8. Hu CM, Fang RH, Luk BT, Zhang L. Nanoparticle-detained toxins for safe and effective vaccination. Nat Nanotechnol. 2013;8(12): 933-938.

9. Hu CM, Fang RH, Copp J, Luk BT, Zhang L. A biomimetic nanosponge that absorbs pore-forming toxins. Nat Nanotechnol. 2013;8(5): 336-340.

10. Parodi A, Quattrocchi N, van de Ven AL, et al. Synthetic nanoparticles functionalized with biomimetic leukocyte membranes possess cell-like functions. Nat Nanotechnol. 2013;8(1):61-68.

11. Fang RH, Hu CM, Luk BT, et al. Cancer cell membrane-coated nanoparticles for anticancer vaccination and drug delivery. Nano Lett. 2014;14(4):2181-2188.

12. Hu CM, Zhang L, Aryal S, Cheung C, Fang RH, Zhang L. Erythrocyte membrane-camouflaged polymeric nanoparticles as a biomimetic delivery platform. Proc Natl Acad Sci U S A. 2011;108(27):10980-10985.

13. Luk BT, Hu C-MJ, Fang RH, et al. Interfacial interactions between natural RBC membranes and synthetic polymeric nanoparticles. Nanoscale. 2014;6(5):2730-2737.

14. Hu CM, Fang RH, Luk BT, et al. 'Marker-of-self' functionalization of nanoscale particles through a top-down cellular membrane coating approach. Nanoscale. 2013;5(7):2664-2668.

15. Gao W, Hu CM, Fang RH, Luk BT, Su J, Zhang L. Surface functionalization of gold nanoparticles with red blood cell membranes. Adv Mater. 2013;25(26):3549-3553.

16. Piao JG, Wang L, Gao F, You YZ, Xiong Y, Yang L. Erythrocyte membrane is an alternative coating to polyethylene glycol for prolonging the circulation lifetime of gold nanocages for photothermal therapy. ACS Nano. 2014;8(10):10414-10425. 
17. Li LL, Xu JH, Qi GB, Zhao X, Yu F, Wang H. Core-shell supramolecular gelatin nanoparticles for adaptive and "on-demand" antibiotic delivery. ACS Nano. 2014;8(5):4975-4983.

18. Knop K, Hoogenboom R, Fischer D, Schubert US. Poly(ethylene glycol) in drug delivery: pros and cons as well as potential alternatives. Angew Chem Int Ed Engl. 2010;49(36):6288-6308.

19. Ishida T, Maeda R, Ichihara M, Irimura K, Kiwada H. Accelerated clearance of PEGylated liposomes in rats after repeated injections. $J$ Control Release. 2003;88(1):35-42.

20. Ishida $\mathrm{T}$, Ichihara $\mathrm{M}$, Wang X, et al. Injection of PEGylated liposomes in rats elicits PEG-specific IgM, which is responsible for rapid elimination of a second dose of PEGylated liposomes. J Control Release. 2006;112(1):15-25.

21. Armstrong JK, Hempel G, Koling S, et al. Antibody against poly(ethylene glycol) adversely affects PEG-asparaginase therapy in acute lymphoblastic leukemia patients. Cancer. 2007;110(1):103-111.

22. Davis ME, Chen ZG, Shin DM. Nanoparticle therapeutics: an emerging treatment modality for cancer. Nat Rev Drug Discov. 2008; 7(9):771-782.

23. Peer D, Karp JM, Hong S, Farokhzad OC, Margalit R, Langer R. Nanocarriers as an emerging platform for cancer therapy. Nat Nanotechnol. 2007;2(12):751-760

24. Wagner V, Dullaart A, Bock AK, Zweck A. The emerging nanomedicine landscape. Nat Biotechnol. 2006;24(10):1211-1217.

25. Muzykantov VR. Drug delivery by red blood cells: vascular carriers designed by mother nature. Expert Opin Drug Deliv. 2010;7(4): 403-427.

26. Murciano JC, Medinilla S, Eslin D, Atochina E, Cines DB, Muzykantov VR. Prophylactic fibrinolysis through selective dissolution of nascent clots by tPA-carrying erythrocytes. Nature Biotech. 2003;21(8):891-896.

27. Wang Q, Cheng H, Peng H, Zhou H, Li PY, Langer R. Non-genetic engineering of cells for drug delivery and cell-based therapy. Adv Drug Deliv Rev. 2015;91:125-140.

28. Fang $\mathrm{RH}, \mathrm{Hu} \mathrm{CM}$, Chen $\mathrm{KN}$, et al. Lipid insertion enables targeting functionalization of erythrocyte membrane-cloaked nanoparticles. Nanoscale. 2013;5(19):8884-8888.

29. Sha H, Zou Z, Xin K, et al. Tumor-penetrating peptide fused EGFR single-domain antibody enhances cancer drugpenetration into $3 \mathrm{D}$ multicellular spheroids and facilitates effective gastric cancer therapy. J Control Release. 2015;200:188-200.

30. Sha H, Li R, Bian X, et al. A tumor-penetrating recombinant protein antiEGFR-iRGD enhance efficacy of paclitaxel in 3D multicellular spheroids and gastric cancer in vivo. Eur J Pharm Sci. 2015;77:60-72.

31. Zhao BX, Zhao Y, Huang Y, et al. The efficiency of tumor-specific $\mathrm{pH}$ responsive peptide-modified polymeric micelles containing paclitaxel. Biomaterials. 2012;33(8):2508-2520.

32. Shi G, Mukthavaram R, Kesari S, Simberg D. Distearoyl anchor-painted erythrocytes with prolonged ligand retention and circulation properties in vivo. Adv Healthc Mater. 2014;3(1):142-148.

33. Danhier F, Lecouturier N, Vroman B, et al. Paclitaxel-loaded PEGylated PLGA-based nanoparticles: in vitro and in vivo evaluation. J Control Release. 2009;133(1):11-17.
34. Liang L, Lin SW, Dai W, et al. Novel cathepsin B-sensitive paclitaxel conjugate: higher water solubility, better efficacy and lower toxicity. J Control Release. 2012;160(3):618-629.

35. Sugahara KN, Scodeller P, Braun GB, et al. A tumor-penetrating peptide enhances circulation-independent targeting of peritoneal carcinomatosis. J Control Release. 2015;212:59-69.

36. Elzoghby AO, Samy WM, Elgindy NA. Albumin-based nanoparticles as potential controlled release drug delivery systems. J Control Release. 2012;157(2):168-182.

37. Ding D, Tang X, Cao X, et al. Novel self-assembly endows human serum albumin nanoparticles with an enhanced antitumor efficacy. AAPS PharmSciTech. 2014;15(1):213-222.

38. Lu J, Chuan X, Zhang H, et al. Free paclitaxel loaded PEGylated-paclitaxel nanoparticles: preparation and comparison with other paclitaxelsystems in vitro and in vivo. Int J Pharm. 2014;471(1-2):525-535.

39. Zhou J, Zhao WY, Ma X, et al. The anticancer efficacy of paclitaxel liposomes modified with mitochondrialtargeting conjugate in resistant lung cancer. Biomaterials. 2013;34(14):3626-3638.

40. Shin HC, Alani AW, Rao DA, Rockich NC, Kwon GS. Multi-drug loaded polymeric micelles for simultaneous delivery of poorly soluble anticancer drugs. J Control Release. 2009;140(3):294-300.

41. Gaucher G, Dufresne MH, Sant VP, Kang N, Maysinger D, Leroux JC. Block copolymer micelles: preparation, characterization and application in drug delivery. J Control Release. 2005;109(1-3):169-188.

42. Zhu Z, Li Y, Li X, et al. Paclitaxel-loaded poly(N-vinylpyrrolidone)b-poly(e-caprolactone) nanoparticles: preparation and antitumor activity in vivo. J Control Release. 2010;142(3):438-446.

43. $\mathrm{Hu} \mathrm{Q}, \mathrm{Gao} \mathrm{X}, \mathrm{Gu} \mathrm{G}$, et al. Glioma therapy using tumor homing and penetrating peptide-functionalized PEG-PLA nanoparticles loaded with paclitaxel. Biomaterials. 2013;34(22):5640-5650.

44. Danhier F, Vroman B, Lecouturier N, et al. Targeting of tumor endothelium by RGD-grafted PLGA-nanoparticles loaded with paclitaxel. J Control Release. 2009;140(2):166-173.

45. Bale AS, Barone S Jr, Scott CS, Cooper GS. A review of potential neurotoxic mechanisms among three chlorinated organic solvents. Toxicol Appl Pharmacol. 2011;255(1):113-126.

46. Xu P, Wang R, Wang X, Ouyang J. Recent advancements in erythrocytes, platelets, and albumin as delivery systems. Onco Targets Ther. 2016;9:2873-2884.

47. Sun Y, Su J, Liu G, et al. Advances of blood cell-based drug delivery systems. Eur J Pharm Sci. 2017;96:115-128.

48. Tzounakas VL, Karadimas DG, Papassideri IS, Seghatchian J, Antonelou MH. Erythrocyte-based drug delivery in transfusion medicine: wandering questions seeking answers. Transfus Apher Sci. 2017 56(4):626-634.

49. Harisa GI, Badran MM, Alanazi FK. Erythrocyte nanovesicles: biogenesis, biological roles and therapeutic approach: erythrocyte nanovesicles. Saudi Pharm J. 2017;25(1):8-17.

50. Tan S, Wu T, Zhang D, Zhang Z. Cell or cell membrane-based drug delivery systems. Theranostics. 2015;5(8):863-881.

51. Zhou H, Fan Z, Lemons PK, Cheng H. A facile approach to functionalize cell membrane-coated nanoparticles. Theranostics. 2016; 6(7):1012-1022.
International Journal of Nanomedicine

\section{Publish your work in this journal}

The International Journal of Nanomedicine is an international, peerreviewed journal focusing on the application of nanotechnology in diagnostics, therapeutics, and drug delivery systems throughout the biomedical field. This journal is indexed on PubMed Central, MedLine, CAS, SciSearch $®$, Current Contents $\AA /$ Clinical Medicine,

\section{Dovepress}

Journal Citation Reports/Science Edition, EMBase, Scopus and the Elsevier Bibliographic databases. The manuscript management system is completely online and includes a very quick and fair peer-review system, which is all easy to use. Visit http://www.dovepress.com/ testimonials.php to read real quotes from published authors. 